\title{
Diffuse Speckle Contrast Analysis (DSCA) for Deep Tissue Blood Flow Monitoring
}

\author{
Kijoon LEE*
}

\begin{abstract}
Assessing blood flow, as well as blood volume and oxygen saturation, is of utmost importance for health monitoring. Assessing microcirculation, as opposed to macrocirculation, is especially important for assessing the local tissue viability. Many optics-based modalities have been developed recently such as laser speckle contrast imaging (LSCI), laser Doppler flowmetry (LDF), diffuse correlation spectroscopy (DCS), and diffuse speckle contrast analysis (DSCA), which all share the advantage of non-invasive measurement of blood flow using non-ionizing radiation. This paper offers a review of these modalities centered around DSCA, which was developed most recently and is considered one of the fastest and most affordable deep tissue perfusion probes.
\end{abstract}

Keywords: diffuse optics, diffuse speckle contrast analysis, blood flow monitoring, perfusion.

Adv Biomed Eng. 9: pp. 21-30, 2020.

\section{Introduction}

After the two original papers were published in tandem in 2013 [1,2], diffuse speckle contrast analysis (DSCA) has been extensively studied both theoretically [10-12] and experimentally [55,59]. Due to its simplicity, cost-effectiveness, and real-time operation, its clinical application is expanding fast $[5,7,8]$.

Blood flow is probably the most important physiological variable relevant to personal health. A human body can be thought of as a conglomerate of tens of trillions of individual cells, whose life being maintained by continuous supply of oxygen and nutrients, and by constant waste collection, all made possible by constantly flowing blood. Therefore, assessing both macroscopic and microscopic blood flow level at any given location and monitoring it in real-time would be of tremendous importance in health management. Local microcirculation, in particular, is very important for tissue viability and wound healing, and this is the area where DSCA is very successfully employed. DSCA addresses the microcirculation level of a given volume of tissue in non-invasive, cost-effective way using speckle contrast, which contains information on scatterers' movement along the way the photon propagates from source to detector.

Before the introduction of DSCA, there were two

Received on May 19, 2019; revised on September 29, 2019; accepted on October 23, 2019.

* College of Transdisciplinary Studies, Daegu-Gyeongbuk Institute of Science and Technology (DGIST), 333 Techno Jungang-daero, Daegu, Korea.

E-mail:kjlee@dgist.ac.kr excellent light-based blood flow measurement modalities, one for shallow depth and the other for deep tissue, which we call laser speckle contrast imaging (LSCI) and diffuse correlation spectroscopy (DCS), respectively. Here, the shallow and deep region refers to the case where the detected photons are mostly single scattered and multiple scattered, respectively. Transport length (inverse of reduced scattering coefficient) can serve as a good threshold between shallow and deep tissue. As we shall see more deeply in later chapters, LSCI is a fantastic blood flow imaging modality equipped with a simple image-processing algorithm which turns a feature-less laser speckle image of a tissue surface into a high-contrast blood-flow image. DCS, on the other hand, is a deep-tissue blood flow monitoring modality using a point source and a point detector, which requires more sophisticated instrumentation and model-fitting process based on diffusing wave spectroscopy (DWS) methodology. These two modalities seemed to be totally different, in the sense that they are probing totally different depth of given tissue and that their instrumentation and analysis were totally different.

Renzhe Bi and others, however, have noted on the similarity between these two modalities - both modalities are measuring the fluctuation of laser speckle, out of which the blood flow information can be extracted. It is just that LSCI looks at mostly specular reflection (or single scattering), whereas DCS looks at diffuse reflection (or multiple scattering) of incoming light. This realization has led the group to develop theoretical model for applying LSCI methodology to diffuse reflected light, and compare with experimental results. The experimental result came out beautifully, and they named it diffuse 
speckle contrast analysis (DSCA), as the modality analyzes speckle contrast resulting from diffuse photons. In Fig. 1 below, the experimental schematic for DCS and DSCA are shown respectively, for immediate comparison. When compared to DCS, DSCA was shown to have much higher time resolution as shown in Fig. 2, and cost-effective as well.

DSCA has two different sub-categories depending on how the speckle contrast is obtained. Spatial DSCA (sDSCA) makes use of statistics among multiple pixels on a single CCD frame, whereas temporal DSCA (tDSCA) makes use of statistics among multiple frame values on a single CCD pixel. These two approaches are identical as long as the probing sample behaves ergodically, and one can choose between them according to their convenience. SDSCA is better for higher temporal resolution, and tDSCA is better for concurrent measurement of multiple channel probe.

In this review paper, a theoretical aspect and instru-

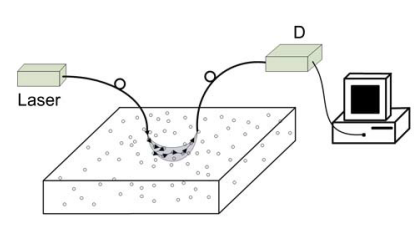

(a)

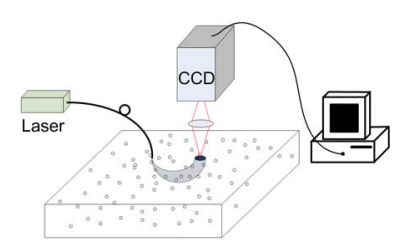

(b)
Fig. 1 Schematic of DCS vs DSCA. In DCS (a), the fluctuating intensity of a single speckle is detected by using singlemode fiber, whereas in DSCA (b), a finite region is imaged by using a CCD camera so that speckle contrast can be obtained for a given exposure time [1].

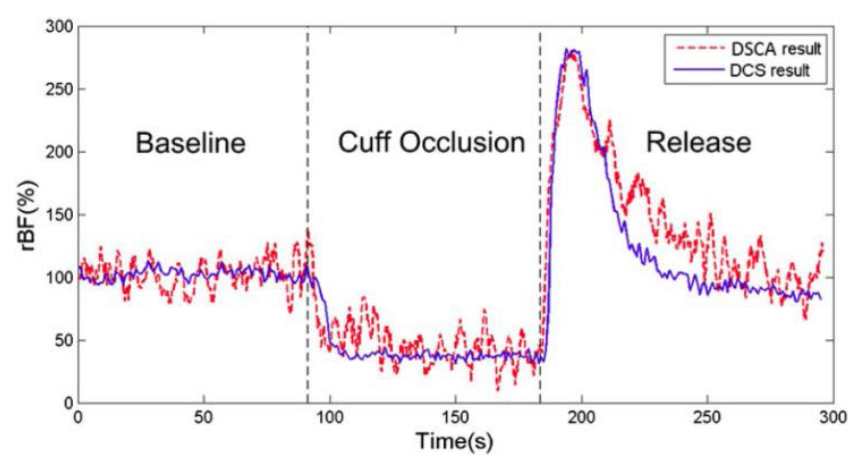

Fig. 2 Blood flow index (BFI) measured concurrently by DCS and by DSCA, during upper limb arterial occlusion protocol, normalized at baseline value. DSCA result shows better time resolution, and hence shows richer content. The fluctuating signal in DSCA is low frequency oscillation (LFO) in capillary flow, which cannot be obtained when time resolution is not sufficiently high. [1] mentational aspect of DSCA will be reviewed, and comparison with other blood flow monitoring modalities will be given. Hopefully this paper will help to deepen the understanding of light-based flow monitoring technology in general.

\section{Theoretical Aspect}

What is speckle? - When laser light is reflected off of a rough surface such as paper or wall, we normally observe a granular pattern on the reflecting surface. This 'speckle' pattern is due to the interference among laser beams with slightly different light paths, and normally regarded as an undesirable property of laser light and sometimes people use optical diffuser in order to remove speckle pattern from their images. But speckles actually contain plenty of information on the reflecting surface and/or possible flow of scatterers underneath the surface. Speckles formed by static scatterers will be stationary, and those formed by moving scatterers will be fluctuating in time. The dynamic part of this speckle image can be exploited to assess the flow level of scatterers. Simply speaking, the higher the flow, the lower the speckle contrast for a given camera exposure time.

What is speckle contrast? - Speckle contrast is a measure of the speckle visibility. If you can clearly see the speckle pattern with clear distinction between bright region and dark region, you have a high speckle contrast. Mathematically speaking, the speckle contrast $K$ is defined as below:

$$
K=\frac{\sigma_{s}}{\langle I\rangle}
$$

where $\sigma_{s}$ is the intensity standard deviation among distributed pixels and $\langle I\rangle$ is the mean value of those pixel intensities. This quantity can be calculated for any ensemble of pixels, distributed over either space or time.

When the exposure time $T$ is given, speckle contrast $K$ is given by following expression:

$$
K(T)=\frac{2 \beta}{T} \int_{0}^{T}\left(1-\frac{\tau}{T}\right)\left[g_{1}(\tau)\right]^{2} d \tau
$$

where $g_{1}(\tau)$ is a normalized field autocorrelation function with respect to delay time $\tau$, and $\beta$ is a constant determined by the polarization state and the ratio between detector size and speckle size. The equation above can be thought of as a weighted integral of squared field autocorrelation function, where the weighting function $1-$ $\tau / T$ decreases linearly towards zero value with increasing $\tau$. Thus, $K(T)$ does not reflect the shape of $g_{1}(\tau)$ very well if the decay constant $\tau_{c}$ of $g_{1}(\tau)$ is either too large or too small compared to the exposure time $T$. We will look more closely on this behavior for two cases of $g_{1}(\tau)$ below, one for single scattering and the other for multiple scattering. 


\section{Single scattering case}

When a wide field illumination and wide field imaging is used, light that reaches CCD pixel has undergone mostly single scattering from the sample, and the normalized field autocorrelation is given as below:

$$
g_{1}(\tau)=e^{-\frac{\tau}{\tau_{c}}}
$$

where $\tau_{c}$ is a decay constant which is related to the scatterer's movement. The faster the scatterers move, the smaller $\tau_{c}$ is. When this expression is plugged into the expression of speckle contrast, we have

$$
K=\sqrt{\beta\left\{\frac{\tau_{c}}{T}+\frac{\tau_{c}^{2}}{2 T^{2}}\left[\exp \left(-\frac{2 T}{\tau_{c}}\right)-1\right]\right\}} .
$$

Thus, for a given exposure time $T, K$ is a monotonously increasing function of $\tau_{c}$ so an image of $K$ can reveal the scatterer's movement in inverse sense, because faster movement means smaller $\tau_{c}$ value. The slope between $K$ and $\tau_{c}$, a.k.a. the sensitivity, is not uniform, however, and it is known that highest sensitivity can be achieved for $\tau_{c} \approx T$. Recently developed multi-exposure speckle imaging (MESI) addresses this non-uniform sensitivity issue, and will be covered in later chapters.

\section{Multiple scattering case}

For multiple scattering case, a different autocorrelation function needs to be used. The unnormalized field autocorrelation function satisfies the correlation diffusion equation written below $[60,62]$ :

$$
\begin{aligned}
& \left(-\frac{1}{3 \mu_{s}^{\prime}} \nabla^{2}+\mu_{a}+\frac{1}{3} \alpha \mu_{s}^{\prime} k_{0}^{2}\left\langle\Delta r^{2}(\tau)\right\rangle\right) G_{1}(\vec{r}, \tau) \\
& =S(\vec{r})
\end{aligned}
$$

where $\alpha$ is the volume fraction of moving scatterers, $\mu_{a}$ is absorption coefficient, $\mu_{s}^{\prime}$ is reduced scattering coefficient, $\left\langle\Delta r^{2}(\tau)\right\rangle$ is the mean square displacement of dynamic scatterers after delay time $\tau$, and $S(\vec{r})$ is the isotropic source term.

The above correlation diffusion equation can be solved for typical semi-infinite geometry using the Green's function method, and the solution is written below:

$$
\begin{aligned}
& G_{1}(r, \tau) \\
& \quad=\frac{3 \mu_{s}^{\prime}}{4 \pi}\left[\frac{\exp \left(-k_{D}(\tau) r_{1}\right)}{r_{1}}-\frac{\exp \left(-k_{D}(\tau) r_{2}\right)}{r_{2}}\right]
\end{aligned}
$$

where

$$
\begin{aligned}
& k_{D}(\tau)=\sqrt{3 \mu_{s}^{\prime} \mu_{a}+\alpha \mu_{s}^{\prime 2} k_{0}^{2}\left\langle\Delta r^{2}(\tau)\right\rangle}, \\
& r_{1}=\sqrt{r^{2}+z_{0}^{2}}, \\
& r_{2}=\sqrt{r^{2}+\left(z_{0}+2 z_{b}\right)^{2}}, \\
& z_{0}=1 / \mu_{s}^{\prime},
\end{aligned}
$$

$$
z_{b}=\frac{2\left(1-R_{e f f}\right)}{3 \mu_{s}^{\prime}\left(1+R_{e f f}\right)}
$$

where $r$ is the source-detector separation, $z_{b}$ is the extrapolation distance at which the diffuse correlation is assumed to vanish, and $r_{1}$ and $r_{2}$ refer to source-detector distance for real source and image source, respectively. The expression looks quite intricate, but the solution is obtained by simple method of images with extended boundary condition. For further information, see reference [60].

The term $\left\langle\Delta r^{2}(\tau)\right\rangle$ appearing above can be modeled in two different ways depending on the nature of scatterers' movement. If the scatterers move linearly in random direction, we can write $\left\langle\Delta r^{2}(\tau)\right\rangle=V^{2} \tau^{2}$ where $V$ is average speed of scatterers, and if the scatterers move diffusively like in a random walk, we can write $\left\langle\Delta r^{2}(\tau)\right\rangle=$ $6 D_{B} \tau$ where $D_{B}$ is the diffusion coefficient for Brownian motion. The motion of the red blood cells (RBCs) in capillary might look more like that of random flow, but actually the diffusion model works better due to their tumbling motion and most research groups seem to favor $\left\langle\Delta r^{2}(\tau)\right\rangle=6 D_{B} \tau$ over $\left\langle\Delta r^{2}(\tau)\right\rangle=V^{2} \tau^{2}$ for their analysis. Therefore, the blood flow index (BFI) in DCS is normally defined as $\alpha D_{B}$, since one cannot determine $D_{B}$ separately from $\alpha$ as shown in equation (5). Note that a high BFI can result from either a faster blood flow (high $D_{B}$ ) or a larger blood volume (high $\alpha$ ).

When this autocorrelation function for half-space (semi-infinite geometry) diffusive light field is plugged into the expression of $K$, the expression becomes even more complicated. Bi et al. [1,2] has shown by simulation and phantom experiment that $1 / K^{2}$ is proportional to the flow as depicted in Fig. 3, and Liu et al. [10,11] has later published theoretical work which supports this linearity. In diffusion regime, therefore, one can define a blood flow index (BFI) as a quantity that is inversely proportional to the speckle contrast squared.

It should be noted that at the time of its birth, the use

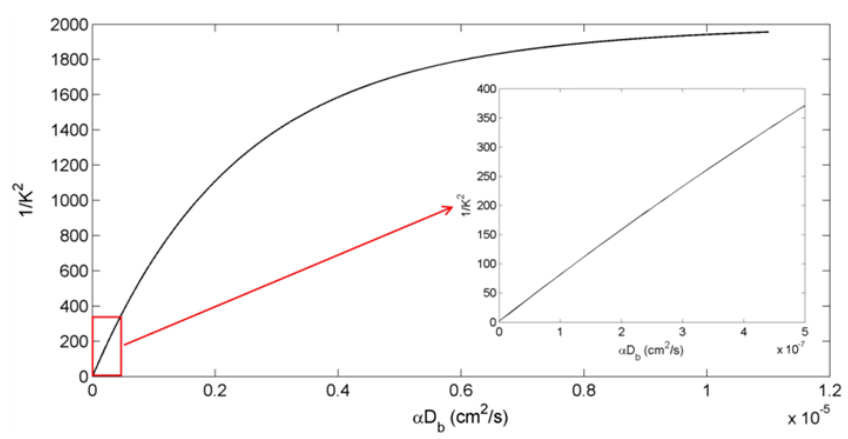

Fig. $31 / K^{2}$ vs $\alpha D_{B}$ graph, The dependence is linear within the physiologically relevant flow range, as shown in the inset. [2] 
of $1 / K^{2}$ as a blood flow index (BFI) of DSCA was rather speculative, but a series of thorough theoretical work has been done by Liu et al $[10,11]$ which supports the validity of $1 / K^{2}$ in diffuse regime. The usefulness of $1 / K^{2}$ in LSCI has been hinted in single-scattering regime before [9] on ad hoc basis, but there was no guarantee that the same formula should work in diffuse (multi-scattering) regime.

Liu et al. [11] has carefully formulated the relationship among speckle contrast $K$, exposure time $T$, source-detector separation $r$, along with diffusion coefficient $D_{B}$ and baseline optical properties (absorption and scattering coefficients), under the condition of $T>\tau_{c}$. That is,

$$
\frac{1}{K^{2}(r, T)}=\frac{1}{\beta}\left(\frac{3 \mu_{s}{ }^{2} k_{0}{ }^{2} \alpha D_{B}}{4 \chi} T+b_{i n}\right)
$$

where $\chi=\frac{1}{2 G_{0}{ }^{2}} \sum_{i=1}^{2} \sum_{j=1}^{2} \frac{(-1)^{i+j}}{r_{i} r_{j}\left(r_{i}+r_{j}\right)^{2}}\left[1+K_{0}\left(r_{i}+r_{j}\right)\right]$ $e^{-K_{0}\left(r_{i}+r_{j}\right)}, K_{0}=\sqrt{3 \mu_{a} \mu_{s}{ }^{\prime}}, G_{0}=4 \pi G_{1}(r, 0) / 3 \mu_{s}{ }^{\prime}$ and $b_{i n}$ is the y-intercept which is shown to be very small.

Therefore, the slope between $1 / K^{2}$ and $T\left(k_{\text {slope }}\right.$, in other words) bears the information on blood flow, and further, $1 / K^{2}$ shows quite linear behavior with blood flow for given exposure time $T$. This justifies the BFI measurement capability of DSCA in relative sense. For absolute measurement of $D_{B}$, one would need to resort to the slope $k_{\text {slope }}$ and incorporate baseline optical properties as well.

\section{Instrumentation}

As the speckle contrast $K$ is defined as a ratio between statistical variables (mean and standard deviation), its instrumentation can be categorized into two regimes depending on how the statistics is applied. When we calculate the mean and standard deviation over multiple pixels in a single frame, we call it spatial DSCA or sDSCA, and when they are calculated over pixel values on different frames for a fixed pixel, we call it temporal DSCA or tDSCA. A diagram shown below (Fig. 4) exemplifies the difference between these two approaches.

\subsection{Spatial DSCA}

Spatial DSCA refers to the kind of DSCA where statistics over different pixels in a single frame are used in order to calculate the speckle contrast. As the $K$ values are obtained for each CCD frame, one does not sacrifice time resolution at all, assuming computation time is negligible. Per frame, one can decide on the appropriate window size as there is a trade-off between SNR and spatial resolution. In traditional LSCI, spatial statistics over a relatively small window of pixels (about $7 \times 7$ pixels) was commonly used and a sliding window was used in order to generate an image of blood vessels. In

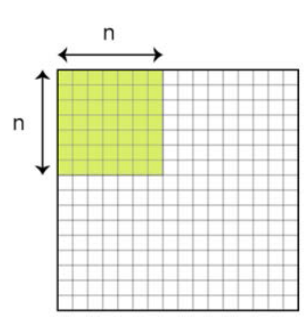

a) SDSCA

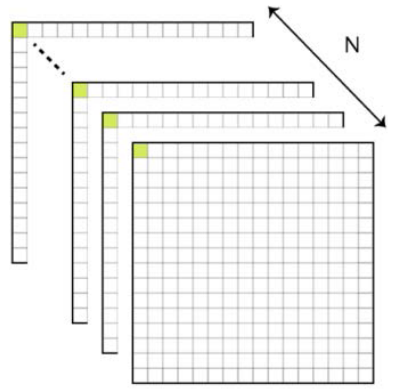

b) TDSCA
Fig. 4 Two different flavors of DSCA. (a) spatial DSCA (sDSCA) uses statistics over $\mathrm{n} \times \mathrm{n}$ pixel window on a single frame and assigns the BFI on the center pixel position of the window. Here a $7 \times 7$ window is shown as an example. (b) temporal DSCA (tDSCA) uses statistics over $N$ frames per fixed pixel.

DSCA, however, one is dealing with diffuse photons and there is no point in using a small number of pixels, as flow imaging is not the main issue in diffuse regime. Hence, one can make use of as many pixels as possible to achieve high signal-to-noise ratio, and as many as $50 \times$ 50 pixels were used in the original paper [1].

\subsection{Temporal DSCA}

There are situations where temporal statistics is favored over spatial statistics. For example, if one wants to maximize the spatial resolution, it would be better to keep each pixel intact and use statistics over some tens of frames to calculate the BFI per pixel. This means we are losing temporal resolution quite drastically, and one would have to use his/her discretion carefully.

It might seem that there is no reason to use temporal statistics for DSCA as one wouldn't require high spatial resolution in DSCA in general, but one particular situation where temporal DSCA is favored is found in multi-channel DSCA. If one wants to put DSCA probe on many locations on a human body and perform a concurrent monitoring, then one obvious way to do that would be to use fiber-based probes and combine the detector fibers together and place them on a single CCD detector. Blood flow information from each channel can be obtained by calculating $K$ for those pixels under the particular detector fiber by temporal DSCA. As one detector fiber will normally cover quite a few pixels, it would be a good practice to bin all the pixels around a single detector fiber in order to increase the SNR. The number of channels one can use is only limited by the area of CCD chip, and one can easily put more than 20 channels snuggly on a single $1 \mathrm{~cm} \times 1 \mathrm{~cm}$ CCD chip.

One technical difficulty of multi-channel tDSCA is the fiber-to-CCD coupling. As one wouldn't want to have 


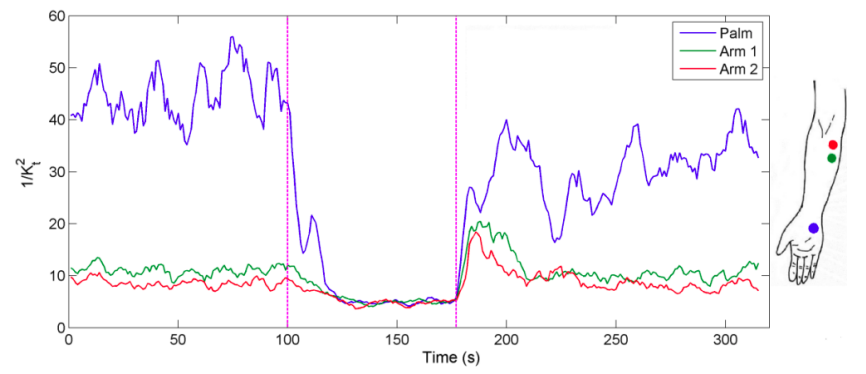

Fig. 5 Concurrent monitoring of BFI on the three different positions on a human arm during cuff occlusion protocol, measured by tDSCA. Similar patterns of low frequency oscillations (LFOs) are observed from closely located probes. The probe positions on lower arm are shown on the right $[2,3]$.

a crosstalk between channels, fibers cannot be placed too closely to each other. Lens coupling between the fiber tip and CCD could be tried as in [6,16], but cross-talk level in this case becomes an issue, as there are many stray lights being produced at the interfaces of optical components. The best way to couple fibers onto a CCD would be to directly put fiber tips on top of the CCD chip. The protection window on CCD should be carefully removed, and fiber bundles should be supported on an opto-mechanical contraption so that its tip can approach the CCD surface without damaging its surface. Careful test needs to be done after installation in order to validate there is indeed a negligible crosstalk between channels.

As exemplified in Fig. 5 above, multi-channel concurrent monitoring of blood flow index enables one to perform a comprehensive study on physiological oscillations of capillary blood flow over a broad area of human body. The three optical probes that share the same laser source and the same CCD have been placed on three different spots on human arm as shown on the right side of Fig. 5. As the blue curve is from the palm tissue where plenty of small muscles reside with dense vasculature, one can see its baseline blood flow is significantly higher than those from arm tissue. The low frequency oscillation (LFO) inherent in microcirculation, caused by slow modulation of sphincters between arteriole and capillary, contains important information regarding vascular health, which in turn relates to tissue viability. It is regretful that not much large-scale multi-channel BFI studies have been seen in the literature yet, although some proof-of-concept work related to angiosome concept does exist [58].

\section{Comparison with other modalities}

The DSCA modality surely did not come into existence out of nothing. For superficial blood flow monitoring, LSCI (or LASCA) and laser Doppler flowmetry (LDF) was already established, and for deep tissue blood flow monitoring, DCS was gaining popularity. Therefore, it would be worthwhile to look into the commonality and differences among the blood-flow monitoring modalities including DSCA. Many parts of the contents are from [4], but some new insights are also included.

\subsection{Comparison with LSCI}

Ever since the clever idea of turning a blurry speckle image into a sharp flow image by a very simple image processing algorithm was presented [61], laser speckle flowmetry has enjoyed a vast amount of attention in biomedical optics [28,29]. Although it was hard to obtain quantitative blood flow measurement from the resulting image of speckle contrast, it has found plenty of application in superficial blood vessel imaging, and there has been a lot of studies aiming to make LSCI a quantitative tools as well [13-15,27,30,34]. Especially, combining LSCI with sidestream dark field (SDF) microscopy has been very successful recently $[35,36]$. There has been great improvement in its instrumentation as well, so that high-speed multi-exposure acquisition is possible [23], deeper penetration becomes possible by using lens array in front of CCD [25], frequency-domain measurement and dual-wavelength measurement is possible [31-33].

DSCA methodology has borrowed a lot of ideas from LSCI, although DSCA is more about measuring averaged value than about imaging the blood flow. Therefore, any development in LSCI can be directly applied to DSCA without too much difficulty. They both have spatial and temporal versions, noise analysis is similar as both of them use CCD as detector, and multi-exposure speckle imaging (MESI) method applied in LSCI was soon applied in DSCA as well.

Although similar in many aspects, there are quite a few differences as well. For example, spatial version of LSCI uses sliding window statistics in order to generate an image of the size similar to original CCD image, whereas sDSCA is only used for non-overlapping windows of pixels which serve as different detector positions, for possible depth-sensitive blood flow probing. Likewise, temporal version of LSCI is used in order to maximize spatial resolution of blood flow image by sacrificing the temporal resolution, whereas tDSCA is mainly used for fiber-based multi-channel flow probes, for possible concurrent measurement on different tissue locations.

\subsection{Comparison with DCS}

Diffuse correlation spectroscopy (DCS) has been one of a kind blood flow monitoring modality which works for deep tissue. It is basically a diffusing wave spectroscopy (DWS) [37] applied to biological sample. Ever since it 
has been known that field autocorrelation function satisfies the same form of differential equation as photon diffusion equation, DCS has found myriads of applications in brain study [46-48], muscle study [43-45], photodynamic therapy monitoring [38,39], diabetic study [40$42]$ and so on, despite its relatively costly hardware and computation-heavy model-fitting process.

A good reference for theoretical understanding of DCS is given by Durduran et al. [60]. The theory starts with radiative transport equation, which reduces into photon diffusion equation in the diffusion regime, where scattering dominates over absorption and source-detector separation is not too small. The field autocorrelation function $G_{1}(\vec{r}, \tau)$ is then shown to satisfy the same photon diffusion equation with flow-dependent absorption term, so that a measurement of $G_{1}(\vec{r}, \tau)$ at certain source-detector separation will give a sense of average blood flow of the tissue region underneath. One caveat has to be given: what is actually being measured from the experiment is the intensity autocorrelation function $G_{2}(\vec{r}, \tau)$. As one cannot directly measure the field autocorrelation function, normally Siegert relation is used in order to convert $G_{2}(\vec{r}, \tau)$ into $G_{1}(\vec{r}, \tau)$, where ergodicity of the whole system is tacitly assumed.

Although DCS has its downside in terms of computational load due to autocorrelation function calculation and model fitting, it is still regarded as the most quantitative means of deep tissue blood flow as it is based on a rigorous model. Even after the development of DSCA and similar speckle-based flow probing modalities, DCS is also improving in both hardware and software [26] and is expected to remain as a viable deep tissue flow probing modality.

\subsection{Comparison with LDF}

Laser Doppler Flowmetry (LDF) is another non-invasive flow monitoring modality that probes a shallow region of tissue. As laser light scattered from moving scatterers will go through a broadening of power spectrum due to Doppler effect, amount of power broadening can be used to assess the level of blood flow.

Although LDF is mainly used for shallow tissue probing and cannot be directly compared with DSCA which is inherently a deep tissue flow measurement modality, LDF can also use a larger source-detector separation as well. This large s-d separation LDF, however, is known to show a non-linear response to the deep tissue flow [5].

A recently developed variation of LDF that exploits the single photon counting detectors [64] is worth noting as it enables more accurate deep tissue flow monitoring by having more sensitive detectors. This single-photon LDF (SP-LDF) has been recently theoretically compared with DCS, and was shown to have a good linear relationship [63]. Although direct comparison between SP-LDF and DSCA has not been done yet, it is expected that there will be a good linear relationship as well, considering the good linearity between DSCA and DCS.

\section{Developing modalities}

In this section, recent advances after the first development of DSCA will be reviewed, with comments on its outlook. It is obvious that DSCA in its current form is far from being an ideal modality for non-invasive deep tissue perfusion monitoring, and further technical and theoretical advancements are anticipated.

As LSCI and DSCA do not provide a quantitative measure of blood flow as is, many attempts have been made in order to make it more quantitative. Multi-exposure speckle imaging (MESI) is one of them, and it calculates the speckle contrast $K$ with several different exposure time $T$ [50,51]. The $K$ values changing with respect to $T$ can be fitted to a model, which gives more quantitative blood flow value.

The principle of MESI was first applied to LSCI [5053], and then to DSCA [10,11]. In case of MESI-DSCA, it has been developed to incorporate multi-distance as well as multi-exposure, in order for simultaneous extraction of multiple parameters [12].

Shortly after the development of DSCA in 2013, on the other hand, a series of model-based modalities were developed claiming better quantification of blood flow. Those are speckle contrast optical tomography (SCOT) and speckle contrast optical spectroscopy (SCOS), both developed from Durduran's group in ICFO [20,21].

For SCOT, the DCS theory has been extended so that one is equipped with the forward model that predicts the speckle contrast $K$ for a given blood flow distribution in deep tissue. They successfully linearized the forward model by using Born approximation, and used it to prove the inverse problem could be solved as well. This modality has been further developed to support high-density probes [22]. For SCOS, the speckle contrast $K$ is measured as a function of either source-detector separation or exposure time, which then is fitted to a model developed based on DCS theory. Their theory includes careful arguments on noise analysis as well, which incorporates shot noise and dark count. SCOS has been made compact using readout noise-free SPAD array recently [24].

Obviously, SCOT and SCOS are more sophisticated approach as they are based on careful theoretical work incorporating diffusing wave spectroscopy (DWS) as well as noise analysis, but the main limitation of model-based approach would be that model-fitting process puts burden on computational resources, making it hard to be applied in real-time application. 
For practical application, MESI-DSCA seems to be a promising modality which provides ease of analysis as well as quantitative measurement, if the clinical situation permits enough data acquisition time for multi-exposure protocol.

Instrument-wise, the principle of DSCA has been applied with simpler hardware, so that even a laser diode and a bare CCD chip were used in contact mode [18]. Also, speckle contrast based 3D reconstruction of blood flow is being pursued, either in contact or non-contact mode $[17,19,49]$.

Also, current trend in medical instrumentation seems to be going in the direction of multi-modality, and DSCA is not an exception. DSCA can be easily integrated with other diffuse optical modalities by sharing their light sources, and even detectors in some cases. A lens-based sDSCA was integrated with diffuse optical spectroscopy device [7], and an off-the-shelf spectrometer has been proposed as a shared detector between DSCA and Diffuse Optical Spectroscopy (DOS) [8].

\section{Clinical applications}

Since its development, DSCA has found applications in many fields. We will mainly focus on clinical application, although there are some applications in industry, including poultry industries [59].

First of all, DSCA finds application in brain study. Brain hemodynamics has crucial meaning not only with diagnostics and treatment monitoring of brain lesions such as hemorrhagic stroke or ischemic stroke, but also with cognitive studies related to localized brain function. Although functional near-infrared spectroscopy (fNIRS) already addresses brain hemodynamics for non-invasive functional studies, fNIRS only measures relative concentrations of oxy-hemoglobin and deoxy-hemoglobin. The missing physiological parameter - blood flow - is very important, as what responds the fastest after a neuronal activation is the local blood flow due to neuro-vascular coupling. Measuring cerebral blood flow will be beneficial 1) for fast detection of brain activity and 2) for estimation of cerebral metabolism of oxygen. Cerebral blood flow can be measured non-invasively either by DCS or DSCA [54], and multi-channel capability of DSCA makes it particularly suitable for cost-effective cerebral blood flow study.

Direct application of DSCA on rat brain was performed by Yeo et al $[55,56]$. Blood flows from multiple regions on brain were concurrently monitored during middle cerebral artery occlusion (MCAO) surgery, showing a physiologically reasonable response.

Another important application of DSCA can be found on periphery, as peripheral vascular health is important in treatment of either burn or diabetes. Burn-re- lated study was performed by [57]. For diabetic foot, which increases incessantly in developed countries, monitoring of blood perfusion on foot tissue with unhealing lesion is very important, especially when vascular surgeons perform balloon angioplasty in order to open up clogged arteries in the leg. As the current gold standard for treatment efficacy assessment is an X-ray angiogram performed 24 hours after the vascular surgery, doctors might lose valuable time in case wrong arteries are opened. Multiple DSCA probes attached on different positions on a patient's foot can help doctors in this case, as it is capable of telling them how much blood perfusion improvement was made after each ballooning episode, and help doctors make real-time decision on surgical strategy.

One important concept for real-time perfusion monitoring during angioplasty is 'angiosome', which refers to a 3-dimensional tissue volume that a particular branch of artery is supplying oxygen and nutrients to. This rather new concept in anatomy and physiology is still debatable, and Yeo et al. has attempted to validate the concept by comparing between inter- and intra-angiosome correlations of low frequency oscillation (LFO) in microcirculation [58]

\section{Conclusion}

Most of the modalities covered within this article fall into the category of diffuse optics, which uses light scattering and interference - linear interaction between photons and tissue. Diffuse optics has been a safe and efficient tool to probe into blood flow as well as blood volume and oxygen saturation in deep tissue. Its hardware and software has been developed tremendously in recent years in the direction of both more sophisticated modeling and better applicability. Among diverse flavors of diffuse optical modalities, DSCA has been recognized as one of the easiest and most cost-effective modalities in terms of applicability due to its simple hardware and straight-forward analysis.

Imagine tens of trillions of red blood cells tumbling through the capillaries to keep your body alive even at this moment. Thanks to the fact that hemoglobin, which by the way has different absorption spectra depending on whether it is oxygenated or not, is confined within a few micron sized red blood cells, one can extract information on blood flow from the light scattering and interference. Another thankful fact is that we have a spectral window of red to near infrared $(650 \mathrm{~nm} \sim 980 \mathrm{~nm})$ where both hemoglobin and water show low absorption, so the lights with this wavelength can reach deep tissue and come back out to surface with valuable information on blood flow.

When fortunes add up, it gives one a sense of confi- 
dence. Blood flow is probably the most important factor in human health, and diffuse optics is probably the best way to non-invasively assess the blood flow, and human health subsequently. The author anticipates that a diffuse optics-based comprehensive, realtime, cost-effective and non-invasive health monitoring system will be developed and routinely used in hospitals within next ten years..

\section{Acknowledgement}

The author acknowledges the support from DGIST through the grant of Educational Innovation Fund.

\section{References}

1. Bi R, Dong J, Lee K. Deep tissue flowmetry based on diffuse speckle contrast analysis. Opt Lett. 38(9), pp. 1401-1403, 2013.

2. Bi R, Dong J, Lee K: Multi-channel deep tissue flowmetry based on temporal diffuse speckle contrast analysis. Opt Express. 21(19), pp. 22854-22861, 2013.

3. Lee K, Bi R, Dong J: Fast and affordable diffuse optical deep-tissue flowmetry. Opt Photon News. 24(12), pp. 32-32, 2013.

4. Bi R, Dong J, Poh CL, Lee K: Optical methods for blood perfusion measurement-theoretical comparison among four different modalities. JOSA A. 32(5), pp. 860-866, 2015.

5. Dong J, Bi R, Lee K: Deep tissue hemodynamic monitoring using diffuse optical probes. In Frontiers in Biophotonics for Translational Medicine (pp. 135-159). Springer, Singapore, 2016.

6. Yeo C, Song C: Diffuse speckle contrast analysis with novel fiber-lens detection. In Optical Tomography and Spectroscopy of Tissue XII (Vol. 10059, p. 1005904). International Society for Optics and Photonics, 2017.

7. Seong M, Phillips Z, Mai PM, Yeo C, Song C, Lee K, Kim JG: Simultaneous blood flow and blood oxygenation measurements using a combination of diffuse speckle contrast analysis and near-infrared spectroscopy. J Biomed Opt. 21(2), 027001, 2016.

8. Seong M, Mai PM, Lee K, Kim JG: Simultaneous blood flow and oxygenation measurements using an off-the-shelf spectrometer. Chinese Opt Lett. 16(7), 071701, 2018.

9. Cheng H, Duong TQ: Simplified laser-speckle-imaging analysis method and its application to retinal blood flow imaging. Opt Lett. 32(15), pp. 2188-2190, 2007.

10. Liu J, Zhang H, Shen Z, Lu J, Ni X: Quantitatively assessing flow velocity by the slope of the inverse square of the contrast values versus camera exposure time. Opt Express. 22(16), pp. 1932719336, 2014.

11. Liu J, Zhang H, Lu J, Ni X, Shen Z: Quantitative model of diffuse speckle contrast analysis for flow measurement. J Biomed Opt. 22(7), 076016, 2017.

12. Liu J, Zhang H, Lu J, Ni X, Shen Z: Simultaneously extracting multiple parameters via multi-distance and multi-exposure diffuse speckle contrast analysis. Biomed Opt Express. 8(10), pp. 4537-4550, 2017.

13. Khaksari K, Kirkpatrick SJ: Laser speckle modeling and simulation for biophysical dynamics: influence of sample statistics. J Biomed Photon Eng. 3(4), 040302, 2017.

14. Humeau-Heurtier A, Abraham P, Mahe G: Linguistic analysis of laser speckle contrast images recorded at rest and during biological zero: comparison with laser Doppler flowmetry data. IEEE
Trans Med Imaging. 32(12), pp. 2311-2321, 2013.

15. Vaz PG, Humeau-Heurtier A, Figueiras E, Correia C, Cardoso J: Effect of static scatterers in laser speckle contrast imaging: an experimental study on correlation and contrast. Phys Med Biol. 63(1), 015024, 2017.

16. Seong M, Phillips Z, Mai PM, Yeo C, Song C, Lee K, Kim JG: Simultaneous blood flow and blood oxygenation measurements using a combination of diffuse speckle contrast analysis and near-infrared spectroscopy. J Biomed Opt. 21(2), 027001, 2016.

17. Huang C, Irwin D, Lin Y, Shang Y, He L, Kong W, Luo J, Yu, G: Speckle contrast diffuse correlation tomography of complex turbid medium flow. Med Phys. 42(7), pp. 4000-4006, 2015.

18. Huang C, Seong M, Morgan JP, Mazdeyasna S, Kim JG, Hastings JT, Yu G: Low-cost compact diffuse speckle contrast flowmeter using small laser diode and bare charge-coupled-device. J Biomed Opt. 21(8), 080501, 2016.

19. Huang C, Irwin D, Zhao M, Shang Y, Agochukwu N, Wong L, Yu G: Noncontact 3-D speckle contrast diffuse correlation tomography of tissue blood flow distribution. IEEE Trans Med Imaging. 36(10), pp. 2068-2076, 2017.

20. Varma HM, Valdes CP, Kristoffersen AK, Culver JP, Durduran T: Speckle contrast optical tomography: A new method for deep tissue three-dimensional tomography of blood flow. Biomed Opt Express. 5(4), pp. 1275-1289, 2014.

21. Valdes CP, Varma HM, Kristoffersen AK, Dragojevic T, Culver JP, Durduran T: Speckle contrast optical spectroscopy, a non-invasive, diffuse optical method for measuring microvascular blood flow in tissue. Biomed Opt Express. 5(8), pp. 2769-2784, 2014.

22. Dragojević T, Varma HM, Hollmann JL, Valdes CP, Culver JP, Justicia C, Durduran T: High-density speckle contrast optical tomography (SCOT) for three dimensional tomographic imaging of the small animal brain. Neuroimage. 153, pp. 283-292, 2017.

23. Dragojević T, Bronzi D, Varma HM, Valdes CP, Castellvi C, Villa F, Tosi A, Justica C, Zappa F, Durduran T: High-speed multi-exposure laser speckle contrast imaging with a single-photon counting camera. Biomed Opt Express. 6(8), pp. 2865-2876, 2015.

24. Dragojević T, Hollmann JL, Tamborini D, Portaluppi D, Buttafava M, Culver JP, Villa F, Durduran T: Compact, multi-exposure speckle contrast optical spectroscopy (SCOS) device for measuring deep tissue blood flow. Biomed Opt Express. 9(1), pp. 322334, 2018.

25. Abookasis D, Moshe T: Feasibility study of hidden flow imaging based on laser speckle technique using multiperspectives contrast images. Opt Lasers Eng. 62, pp. 38-45, 2014.

26. Tivnan M, Gurjar R, Wolf D, Vishwanath K: High frequency sampling of TTL pulses on a Raspberry Pi for diffuse correlation spectroscopy applications. Sensors. 15(8), pp. 19709-19722, 2015.

27. Duncan DD, Kirkpatrick SJ: Can laser speckle flowmetry be made a quantitative tool? JOSA A. 25(8), pp. 2088-2094, 2008.

28. Boas DA, Dunn AK: Laser speckle contrast imaging in biomedical optics. J Biomed Opt. 15(1), 011109, 2010.

29. Zakharov P, Völker AC, Wyss MT, Haiss F, Calcinaghi N, Zunzunegui C, Buck A, Scheffold F, Weber B: Dynamic laser speckle imaging of cerebral blood flow. Opt Express. 17(16), pp. 1390413917,2009 
30. Briers D, Duncan DD, Hirst ER, Kirkpatrick SJ, Larsson M, Steenbergen W, Stromberg T, Thompson OB: Laser speckle contrast imaging: theoretical and practical limitations. J Biomed Opt. 18(6), 066018, 2013.

31. Li H, Liu Q, Lu H, Li Y, Zhang HF, Tong S: Directly measuring absolute flow speed by frequency-domain laser speckle imaging. Opt Express. 22(17), pp. 21079-21087, 2014.

32. Vaz PG, Humeau-Heurtier A, Figueiras E, Correia C, Cardoso J: Laser speckle imaging to monitor microvascular blood flow: a review. IEEE Rev Biomed Eng. 9, pp. 106-120, 2016.

33. Abookasis D, Volkov B, Shochat A, Kofman I: Noninvasive assessment of hemodynamic and brain metabolism parameters following closed head injury in a mouse model by comparative diffuse optical reflectance approaches. Neurophotonics. 3(2), 025003, 2016.

34. Ji Y, Chao Z, Zhang Y, Wu Z, Miao P: Effects of scattering particles' concentration in laser speckle contrast imaging. In 2014 7th International Conference on Biomedical Engineering and Informatics (pp. 56-60). IEEE, 2014.

35. Nadort A, Woolthuis RG, van Leeuwen TG, Faber DJ: Quantitative laser speckle flowmetry of the in vivo microcirculation using sidestream dark field microscopy. Biomed Opt Express. 4(11), pp. 2347-2361, 2013.

36. Nadort A, Kalkman K, van Leeuwen TG, Faber DJ: Quantitative blood flow velocity imaging using laser speckle flowmetry. Sci Rep. 6, 25258, 2016.

37. Pine DJ, Weitz DA, Chaikin PM, Herbolzheimer E: Diffusing wave spectroscopy. Phys Rev Lett. 60(12), 1134, 1988.

38. Dong J, Toh HJ, Thong PS, Tee CS, Bi R, Soo KC, Lee K: Hemodynamic monitoring of Chlorin e6-mediated photodynamic therapy using diffuse optical measurements. J Photochem Photobiol B. 140, pp. 163-172, 2014.

39. Thong P, Lee K, Toh HJ, Dong J, Tee CS, Low KP, Chang PH, Bhuvaneswari R, Tan NC, Soo KC: Early assessment of tumor response to photodynamic therapy using combined diffuse opti$\mathrm{cal}$ and diffuse correlation spectroscopy to predict treatment outcome. Oncotarget. 8(12), 19902, 2017.

40. Cheng R, Zhang X, Daugherty A, Shin H, Yu G: Noninvasive quantification of postocclusive reactive hyperemia in mouse thigh muscle by near-infrared diffuse correlation spectroscopy. Appl Opt. 52(30), pp. 7324-7330, 2013.

41. Nakabayashi M, Ono Y: Detection of blood flow speed in shallow and deep tissues using diffuse correlation spectroscopy. Adv Biomed Eng. 6, pp. 53-58, 2017.

42. Ono Y, Esaki K, Takahashi Y, Nakabayashi M, Ichinose M, Lee $\mathrm{K}$ : Muscular blood flow responses as an early predictor of the severity of diabetic neuropathy at a later stage in streptozotocin-induced type I diabetic rats: a diffuse correlation spectroscopy study. Biomed Opt Express. 9(9), pp. 4539-4551, 2018.

43. Yu G, Floyd TF, Durduran T, Zhou C, Wang J, Detre JA, Yodh AG: Validation of diffuse correlation spectroscopy for muscle blood flow with concurrent arterial spin labeled perfusion MRI. Opt Express. 15(3), pp. 1064-1075, 2007.

44. Shang Y, Symons TB, Durduran T, Yodh AG, Yu G: Effects of muscle fiber motion on diffuse correlation spectroscopy blood flow measurements during exercise. Biomed Opt Express. 1(2), pp. 500-511, 2010.

45. Shang Y, Gurley K, Yu G: Diffuse correlation spectroscopy (DCS) for assessment of tissue blood flow in skeletal muscle: recent progress. Anat Physiol. 3(2), 128, 2013.

46. Buckley EM, Cook NM, Durduran T, Kim MN, Zhou C, Choe R, Yu G, Shultz S, Sehgal CM, Licht DJ, Arger PH, Putt ME, Hurt $\mathrm{H}$, Yodh AG: Cerebral hemodynamics in preterm infants during positional intervention measured with diffuse correlation spectroscopy and transcranial Doppler ultrasound. Opt Express. 17(15), pp. 12571-12581, 2009.

47. Kim MN, Durduran T, Frangos S, Edlow BL, Buckley EM, Moss HE, Zhou C, Yu G, Choe R, Maloney-Wilensky E, Wolf RL, Grady S, Greenberg JH, Levine JM, Yodh AG, Detre JA, Kofke A: Noninvasive measurement of cerebral blood flow and blood oxygenation using near-infrared and diffuse correlation spectroscopies in critically brain-injured adults. Neurocritical Care. 12(2), pp. 173-180, 2010.

48. Durduran T, Yodh AG: Diffuse correlation spectroscopy for non-invasive, micro-vascular cerebral blood flow measurement. Neuroimage. 85, pp. 51-63, 2014.

49. Han S, Johansson J, Mireles M, Proctor AR, Hoffman MD, Vella JB, Benoit DSW, Durduran T, Choe R: Non-contact scanning diffuse correlation tomography system for three-dimensional blood flow imaging in a murine bone graft model. Biomed Opt Express. 6(7), pp. 2695-2712, 2015.

50. Parthasarathy AB, Tom WJ, Gopal A, Zhang X, Dunn AK: Robust flow measurement with multi-exposure speckle imaging. Opt Express. 16(3), pp. 1975-1989, 2008.

51. Parthasarathy AB, Kazmi SMS, Dunn AK: Quantitative imaging of ischemic stroke through thinned skull in mice with Multi Exposure Speckle Imaging. Biomed Opt Express. 1(1), pp. 246$259,2010$.

52. Thompson OB, Andrews MK: Tissue perfusion measurements: multiple-exposure laser speckle analysis generates laser Doppler-like spectra. J Biomed Opt. 15(2), 027015, 2010.

53. Kazmi SMS, Parthasarthy AB, Song NE, Jones TA, Dunn AK: Chronic imaging of cortical blood flow using Multi-Exposure Speckle Imaging. J Cereb Blood Flow Metab. 33(6), pp. 798808, 2013.

54. Buckley EM, Parthasarathy AB, Grant PE, Yodh AG, Franceschini MA: Diffuse correlation spectroscopy for measurement of cerebral blood flow: future prospects. Neurophotonics. 1(1), 011009, 2014.

55. Yeo C, Kim H, Song C: Cerebral Blood Flow Monitoring by Diffuse Speckle Contrast Analysis during MCAO Surgery in the Rat. Curr Opt Photon. 1(5), pp. 433-439, 2017.

56. Yeo CB, Jo WR, Kim HJ, Song C: Diffuse Speckle Contrast Analysis Assisted Intraoperative Blood Flow Monitoring in the Rat Model of Femoral Arterial Occlusion. In 2018 40th Annual International Conference of the IEEE Engineering in Medicine and Biology Society (EMBC) (pp. 858-861). IEEE, 2018.

57. Ragol S, Remer I, Shoham Y, Hazan S, Willenz U, Sinelnikov I, Dronov V, Rosenberg L, Bilenca A: In vivo burn diagnosis by camera-phone diffuse reflectance laser speckle detection. Biomed Opt Express. 7(1), pp. 225-237, 2016.

58. Yeo CB, Lee KJ, Song C: Angiosome based time series analysis of deep tissue perfusion using diffuse speckle contrast analysis. In 2017 39th Annual International Conference of the IEEE Engineering in Medicine and Biology Society (EMBC) (pp. 40544057). IEEE, 2017.

59. Yeo C, Park HC, Lee K, Song C. Avian embryo monitoring during incubation using multi-channel diffuse speckle contrast 
analysis. Biomed Opt Express. 7(1), pp. 93-98, 2016.

60. Durduran T, Choe R, Baker WB, Yodh AG. Diffuse optics for tissue monitoring and tomography. Rep Prog Phys. 73(7), 076701, 2010

61. Fercher AF, Briers JD. Flow visualization by means of single-exposure speckle photography. Opt Commun. 37(5), pp. 326-330, 1981.

62. Cheung C, Culver JP, Takahashi K, Greenberg JH, Yodh AG. In vivo cerebrovascular measurement combining diffuse near-infrared absorption and correlation spectroscopies. Phys Med Biol. 46(8), 2053, 2001

63. Binzoni T, Martelli F. Study on the mathematical relationship existing between single-photon laser-Doppler flowmetry and diffuse correlation spectroscopy with static background. JOSA A. 34(12), pp. 2096-2101, 2017.

64. Binzoni T, Van De Ville D, Sanguinetti B. Time-domain algorithm for single-photon laser-Doppler flowmetry at large interoptode spacing in human bone. Appl Opt. 53(30), pp. 7017-7024, 2014.

\section{Kijoon LeE}

Kijoon LEE has received his B.S and M.S degree in

Physics from Seoul National University, and Ph.D in Physics from Brown University with his work on nonlinear optics in highly scattering medium. After postdoctoral training at the University of Pennsylvania with Prof Arjun Yodh, he joined the Division of Bioengineering at Nanyang Technological University, Singapore, as an Assistant Professor. He is currently an Associate Professor and Dean of College of Transdisciplinary Studies at DGIST (Daegu Gyeongbuk Institute of Science and Technology), Korea. His research interest covers biomedical optics, quantum optics, and physics education. 